\title{
Tumor-Infiltrating T Cells Concurrently Overexpress CD200R with Immune Checkpoints PD-1, CTLA-4, and TIM-3 in Non-Small-Cell Lung Cancer
}

\author{
Yinghan Su ${ }^{a, b}$ Shota Yamazaki ${ }^{a, b}$ Ryo Morisue $^{b, c}$ Jun Suzuki ${ }^{b}$ d \\ Toshiaki Yoshikawa ${ }^{e}$ Tetsuya Nakatsura ${ }^{e}$ Masahiro Tsuboi ${ }^{d}$ Atsushi Ochiai ${ }^{a, f}$ \\ Genichiro Ishiia, b, g \\ a Laboratory of Cancer Biology, Department of Integrated Biosciences, Graduate School of Frontier Sciences, \\ University of Tokyo, Kashiwa, Japan; ${ }^{b}$ Division of Pathology, Exploratory Oncology Research and Clinical Trial \\ Center, National Cancer Center, Kashiwa, Japan; ${ }^{C}$ Department of Hepatobiliary and Pancreatic Surgery, National \\ Cancer Center Hospital East, Kashiwa, Japan; ${ }^{\mathrm{d} D e p a r t m e n t}$ of Thoracic Surgery, National Cancer Center Hospital \\ East, Kashiwa, Japan; ' Division of Cancer Immunotherapy, Exploratory Oncology Research and Clinical Trial Center, \\ National Cancer Center, Kashiwa, Japan; ${ }^{\mathrm{f} E x p l o r a t o r y ~ O n c o l o g y ~ R e s e a r c h ~ a n d ~ C l i n i c a l ~ T r i a l ~ C e n t e r, ~ N a t i o n a l ~ C a n c e r ~}$ \\ Center, Kashiwa, Japan; ${ }^{9}$ Department of Pathology and Clinical Laboratories, National Cancer Center Hospital East, \\ Kashiwa, Japan
}

\section{Keywords}

CD200R · Cytotoxic T-lymphocyte-associated protein 4 . Immune checkpoints · Programmed cell death protein 1 . Tumor-infiltrating leukocytes

\begin{abstract}
Introduction: CD200R has been reported to be the receptor for the immune checkpoint molecule CD200 and can transduce immune-suppressive signals. In this study, we mainly focused on the expression level of CD200R in T cells in pulmonary artery (PA) blood and non-small-cell lung cancer (NSCLC) tumor tissue. Methods: Immune cells were isolated from dissected tumor samples and PA blood of NSCLC patients and analyzed with multiparameter flow cytometry. The co-expression of CD200R with other immune check-
\end{abstract}

points, including programmed cell death protein 1 (PD-1), cytotoxic T-lymphocyte-associated protein 4 (CTLA-4), and T cell immunoglobulin and mucin domain-containing protein 3 (TIM-3), was also investigated. Results: CD200R expression was observed on the surface of approximately $75 \%$ of T cells among tumor-infiltrating leukocytes (TILs). Compared to T cells extracted from TILs, only $55 \%$ of $\mathrm{T}$ cells extracted from PA blood exhibited CD200R expression. Moreover, with higher expression of CD200R, the expression of other immune checkpoints, including PD-1, CTLA-4, and TIM-3, was also increased in tumor-infiltrating $T$ cells compared to $T$ cells in PA blood. Conclusions: Our results showed that those tumors were dominated by T cells expressing CD200R together with other checkpoints, which suggests a phenotypic change after T cell infiltration into the tumor, such as T cell exhaustion.

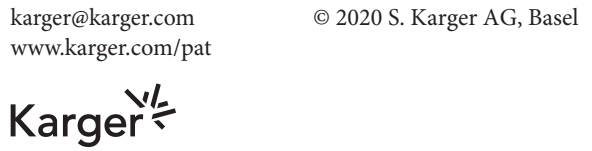




\section{Introduction}

The discovery of immune checkpoints has proven to be one of the most important advancements for cancer therapy development. Immune checkpoints are the key molecules that act as brakes to restore immune homeostasis after diseases, and those molecules are hijacked by cancer cells to inhibit immune activities. The currently best-studied checkpoints are programmed cell death protein 1 (PD-1) and cytotoxic T-lymphocyte-associated protein 4 (CTLA-4). Immune checkpoint inhibitors (ICIs) have already been administered as first-line treatments for advanced-stage non-small-cell lung cancer (NSCLC) patients. Compared with standard chemotherapy, ICIs have led to promising, durable responses and exhibited possible benefits for long-term survival $[1,2]$.

CD200R and its ligand, CD200, have also been reported to be functional immune checkpoint molecules. To date, it is well documented that the interaction between CD200 and CD200R triggers an immune suppression signal that inhibits the RAS/MAPK signal cascades, resulting in a decrease in the production of inflammatory cytokines and an increase in anti-inflammatory cytokine secretion [3-7].

The potential of CD200-CD200R has been widely discussed in the context of diseases or therapies related to the immune system, such as arthritis, infection, and transplantation [8-10]. Many previous studies have claimed that CD200 expressed by melanoma, ovarian cancer, breast cancer, and hepatocellular carcinoma can interact with CD200R, which is a prognostic factor or even a biomarker for cancer stem cells [11-14]. Meanwhile, CD200R was reported as a prognostic factor in lung cancer, and high stromal CD200R expression was strongly connected with non-adenocarcinoma histological types (e.g., squamous cell carcinoma and other subtypes) and also with advanced disease stage [15].

The expression of CD200R is restricted to the surfaces of immune cells, such as monocytes, macrophages, neutrophils, and dendritic cells from the myeloid lineage as well as $\mathrm{T}$ and $\mathrm{B}$ cells from the lymphoid lineage $[3-5,16]$. On the other hand, CD200 is reported to be widely distributed among cells of both hematopoietic and nonhematopoietic origins, such as neurons, endothelial cells, and fibroblasts, as well as among multiple types of cancer [17-20].

Although ICIs have already had great achievement in the treatment of several types of cancer, there is still a proportion of patients who show no response to ICI therapy. Investigations in reliable biomarkers and novel check- points are intensely required in order to enhance the efficacy of ICIs. In this study, we investigated the expression of CD200R in NSCLC tumor tissue and blood taken from dissected lung pulmonary artery (PA) and confirmed its co-expression with three other immune checkpoint molecules.

\section{Materials and Methods}

\section{Tumor-Infiltrating Leukocyte (TIL) Extraction}

Human lung specimens were obtained from the surgically resected lung tissues of NSCLC patients at National Cancer Center Hospital East. The tissue specimens were cut into small pieces with sterilized scissors and tweezers. The minced specimens were then transferred to a 50-mL centrifuge tube with pre-prepared BD Horizon $^{\mathrm{TM}}$ Dri Tumor \& Tissue Dissociation Reagent (BD Biosciences, Inc., Franklin Lakes, NJ, USA) according to the manufacturer's instructions. The mixture was placed in a $37^{\circ} \mathrm{C}$ water bath for 30 min with frequent agitation until the tissue was enzymatically dissociated into a single-cell suspension. The enzymatic reaction was stopped by washing with phosphate-buffered saline (PBS; Sigma-Aldrich, St. Louis, MO, USA) with $1 \%$ bovine serum albumin (Life Technologies, Carlsbad, CA, USA) and ethylenediaminetetraacetic acid (final concentration $2 \mathrm{mM}$; Sigma-Aldrich). The cell suspension was pooled with the stored supernatant and filtered through a $70-\mu \mathrm{m}$ filter (Corning Inc., Corning, NY, USA). After centrifugation, the pelleted samples were treated with BD Pharm Lyse Buffer (BD Biosciences, Inc.) to remove the erythrocytes. After centrifugation, the TIL layers in the tubes were collected and counted with a hemocytometer for downstream assays.

\section{Peripheral Blood Mononuclear Cell (PBMC) Isolation and} Collection

Human peripheral blood was isolated with a heparin (Mochida Pharmaceutical Co., Ltd, Tokyo, Japan)-coated 10-mL syringe from the PA of surgically resected primary lung cancer tissues as previously reported [21]. PBMCs were isolated through centrifugation with BD Vacutainer Evacuated Blood Collection Tubes (BD Biosciences, Inc.) according to the manufacturer's instructions. After centrifugation, the blood mononuclear cell layers in the tubes were collected and counted with a hemocytometer for downstream assays.

\section{T Cell Purification}

The cells obtained from the tumor tissue or PA blood were washed with PBS, quantified, and positively selected by passing through a magnetic-activated cell sorting (MACS) medium column with anti-CD3 antibody-conjugated microbeads (Miltenyi Biotec, Bergisch Gladbach, Germany) according to the manufacturer's protocol. The purity of $\mathrm{CD} 3+\mathrm{T}$ cells was further confirmed with flow cytometry analysis.

\section{Fluorescence-Activated Cell Sorting (FACS) Analysis}

The expression of cell surface antigens was determined by using multicolor immunofluorescence staining and a minimum of 10,000 events were counted with the FACSDiva software (BD Biosciences, Inc.). The conjugated antibodies used were CD3-APC (clone: UCHT1), CD14-APC (clone: 63D3), CD20-APC (clone: 2H7), CD4- 
APC, CD4-Alexa Fluor 700 (clone: RPA-T4), CD8a-APC, CD8aAlexa Fluor 700 (clone: HIT8a), CD200R-PE, CD200R-APC (clone: OX-108), CD279-PE (CD279: PD-1; clone: EH12.2H7), CD152-PE (CD152: CTLA-4; clone: L3D10), and CD366-PE (CD366: T cell immunoglobulin and mucin domain-containing protein 3 [TIM-3]; clone: F38-2E2; BioLegend, San Diego, CA, USA). Fluorescenceconjugated mouse IgG1 (PE, APC \& Alexa Fluor 700; clone: MOPC21) and mouse IgG2b (APC; clone: MPC-11) $\kappa$ isotype Ctrl antibodies (BioLegend) were used as negative controls. Detailed information on all antibodies is provided in online supplementary Table 1 (see www.karger.com/doi/10.1159/000511557 for all online suppl. material). In brief, $100 \mu \mathrm{L}$ of cell suspension (containing $5 \times 10^{5}$ cells) were incubated with $5 \mu \mathrm{L}$ of conjugated antibodies for $1 \mathrm{~h}$ at $4{ }^{\circ} \mathrm{C}$ in the dark. Excess antibodies were removed by washing the cells with PBS (containing 3\% FBS). Then, FACS analysis was performed by using a FACSAria or FACSAccuri C6 plus (BD Biosciences, Inc.). Singlespectrum staining of the aforementioned reagents was used for compensation. The final data analysis was conducted with Flowing Software version 2.51, developed by Perttu Terho (accessed from www. flowingsoftware.com).

Public Database Analysis

To explore whether the results could be generalized to a wider population, an analysis was conducted of a TCGA mRNA expression dataset that included 584 primary lung adenocarcinoma patients by using the cBioPortal for Cancer Genomics web site [22, 23]. Based on the median CD200R1 gene expression, all samples were divided into two groups (the CD200R-high and CD200R-low groups). The correlations between the gene expression of CD200R1 and the gene expression of six different immune checkpoint molecules and another nine immune-related genes were evaluated.

\section{Statistical Analysis}

The significance of differences between the two groups was examined by using Student's $t$ test (paired or unpaired $t$ tests as indicated), and $p<0.05$ was considered statistically significant.

\section{Results}

\section{CD200R Expression Was Observed Mainly in CD3+}

TILs in NSCLC

To confirm whether the expression of CD200R is mainly limited to cell populations of the myeloid lineage, TILs were collected through the enzymatic digestion of dissected tumor specimens for flow cytometry analysis (Fig. 1a; online suppl. Fig. 1A, 1B). Among the infiltrating immune cells, CD3+ T cells accounted for an average of $46.55 \%$ of all extracted cells (Fig. 1b), which was significantly higher than the proportions of monocytes (CD14+; 11.94\%) and B cells (CD20+; 17.61\%). In addition, CD200R was expressed on the surface of $70.52 \%$ of CD3 + T cells (Fig. 1c), and this expression was significantly higher than that of CD14+ cells (46.64\%) and CD20+ cells (61.00\%).

Next, PBMCs were collected from the PA blood of resected lungs, and a similar proportion of CD200R+ cells were observed; of these CD200R+ cells, T cells were most abundant among the extracted immune cells (online suppl. Fig. 1C). In contrast to tumor-infiltrating $\mathrm{T}$ cells, only $55.06 \%$ of the CD3+ T cells extracted from PA blood were found to have CD200R expression (Fig. 1d). To further characterize CD200R+ T cells, MACS was conducted to purify $\mathrm{CD} 3+\mathrm{T}$ cells from all PBMCs to evaluate the expression levels of CD200R on CD4+ and CD8+ T cells (online suppl. Fig. 1D). As Figures $1 \mathrm{~d}$ and e show, both CD4 (73.71\%) and CD8 (66.05\%) $\mathrm{T}$ cells expressed CD200R.

\section{A Higher Proportion of CD200R Expression Was}

Observed in Tumor-Infiltrating T Cells than in

\section{Peripheral Blood T Cells}

To further confirm the differences in CD200R expression between tumor-infiltrating T cells and PA blood-derived T cells, MACS-sorted CD3+ T cells from PA and TILs (online suppl. Fig. 2A, B) were analyzed. Twelve samples of $\mathrm{T}$ cells showed that tumor-infiltrating $\mathrm{T}$ cells were dominated by CD200R+ T cells, compared to peripheral blood T cells (Fig. 2a). Moreover, similar results were obtained in three paired samples of PA blood and tumor tissue (Fig. 2b). On the other hand, CD8+ cells tend to be the main reason for this increase in the CD200R+ population (Fig. 2c), while those numbers in CD4+ cell might differ from sample to sample (Fig. 2d).

\section{Co-Expression of CD200R and Other Immune \\ Checkpoint Molecules}

Given that CD200R is an immune checkpoint molecule which regulates immune cell activity, we investigated cells collected from the paired PA blood and tumor specimens by FACS analysis to determine whether other immune checkpoint molecules may be upregulated and coexpressed with CD200R. An average of 52.02\% of tumorinfiltrating $\mathrm{T}$ cells expressed both PD-1 and CD200R (Fig. 3a, left). In comparison, only an average of $23.21 \%$ of T cells extracted from PA blood expressed both PD-1 and CD200R. Similarly, co-expression of PD-1 and CD200R was also significantly increased among CD4+ (1.61-fold) and CD8+ (1.79-fold) T cells when comparing $\mathrm{T}$ cells derived from PA blood and those derived from tumor tissue (Fig. 3a, right; online suppl. Fig. 3A and 3B, left, and 3C, 3D). For CTLA-4 and TIM-3, both of these checkpoints showed a limited level of expression on the surface of CD3+ T cells derived from PA blood (CTLA-4: 1.19\%; TIM-3: 0.89\%; Fig. 3b and c, left). Among tumorinfiltrating $\mathrm{T}$ cells, CTLA-4 was co-expressed with CD200R on $21.88 \%$ of CD4+ T cells and $20.75 \%$ of CD8 


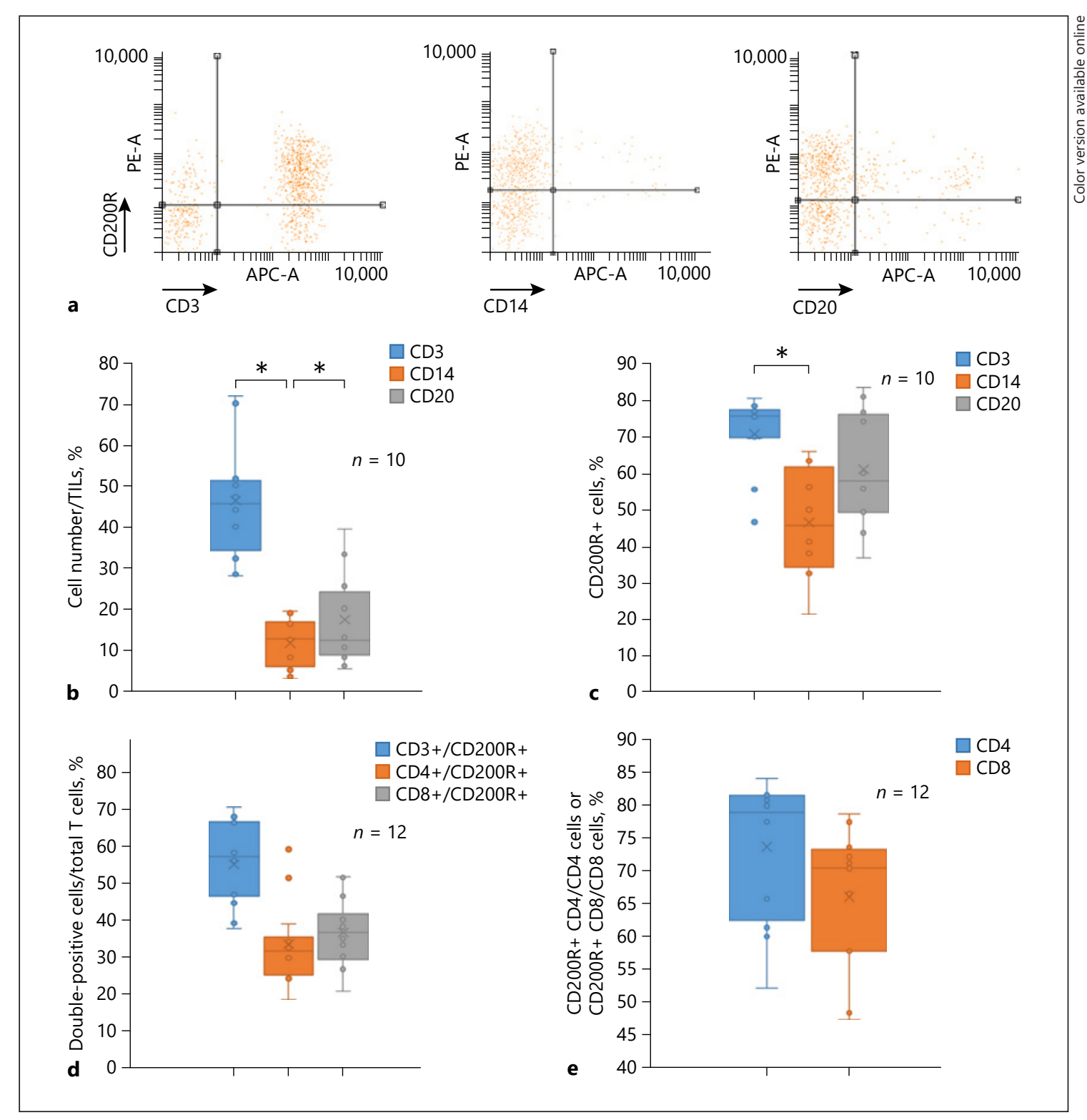

Fig. 1. CD200R-expressing cells within TILs and peripheral blood mononuclear cells extracted from PA blood. a FACS analysis of CD200R+ cells in CD3 (left), CD14 (middle), and CD20 (right) cells. b Proportion of CD $3+$ T cells, CD14+ monocytes, and CD20+ B cells within extracted TILs $(n=10) .{ }^{*} p<0.05$ according to Student's $t$ test. c Proportion of CD200R+ cells within each cell popu-

cells, which was significantly upregulated compared to paired PA samples (Fig. 3b, c; online suppl. Fig. 3A, B, middle and right). Moreover, as Figure $3 \mathrm{c}$ shows, the coexpression of TIM-3 and CD200R was limited to CD8 T cells and showed differences between the PA and TIL samples.

CD200R Co-Expressed with Immune Checkpoints in NSCLC TILs lations $(n=10) . * p<0.05$ according to Student's $t$ test. $\mathbf{d}$ Proportion of T cells with CD3+/CD200R+, CD4+/CD200R+, or CD8+/ CD200R + among all CD3+ T cells extracted from PA blood $(n=$ 12). e Proportion of CD200R + cells within the CD4 and CD8 T cell populations $(n=12)$. FACS, fluorescence-activated cell sorting; PA, pulmonary artery; TILs, tumor-infiltrating leukocytes.

Public Database Analysis Confirmed a High Level of Immune Checkpoint Expression in the CD200R-High Group

To confirm that the co-expression of CD200R and other immune checkpoints can be generalized to a wider population, an analysis of a TCGA lung adenocarcinoma 


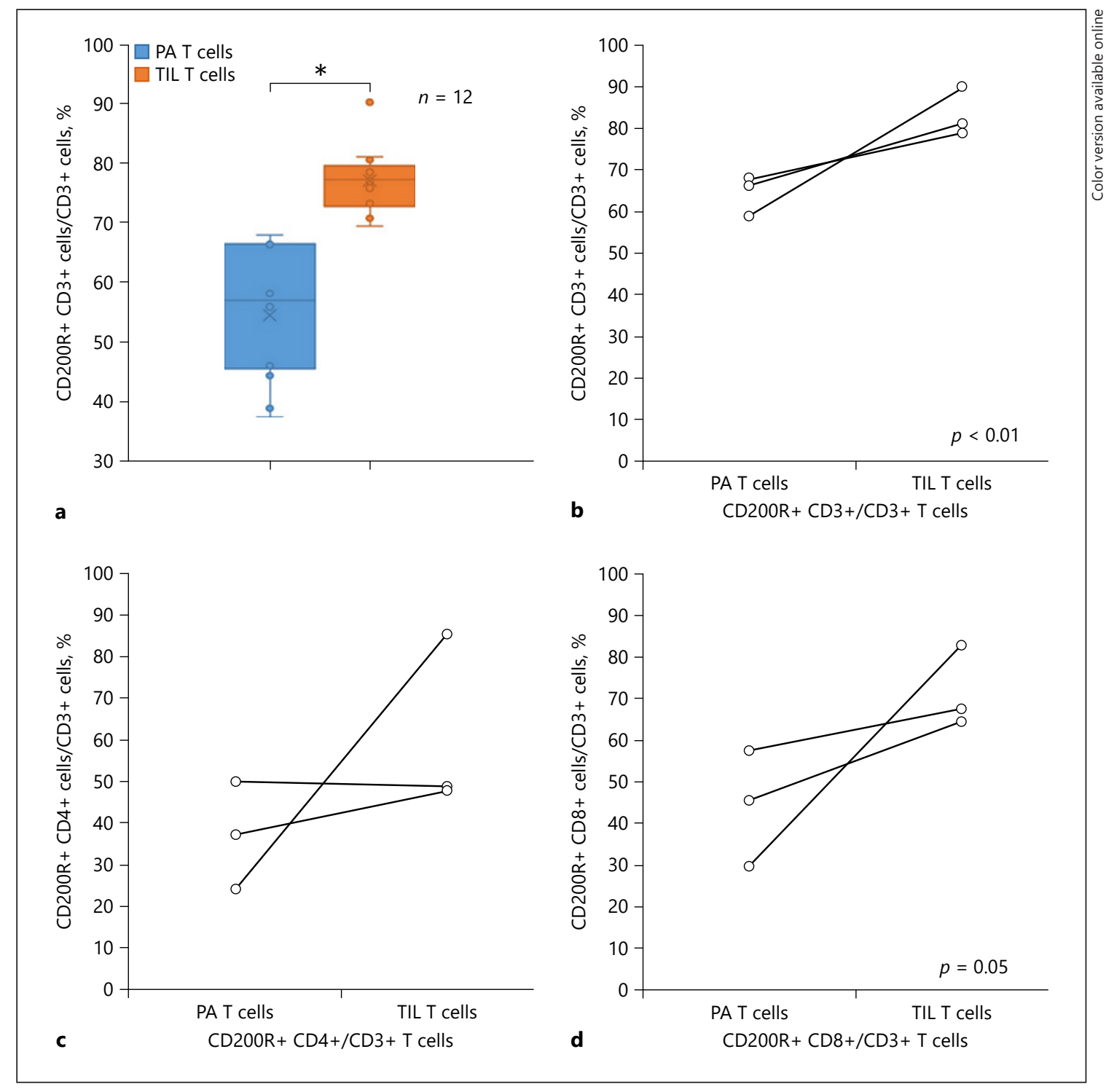

Fig. 2. Comparison of the proportions of CD200R $+\mathrm{T}$ cells in $\mathrm{PA}$ blood and TILs. a Proportion of CD200R + T cells in CD3+ cells from the PA and TIL (unpaired) samples $(n=12) .{ }^{*} p<0.05$ according to unpaired Student's $t$ test. b Proportion of CD200R+ T cells in CD3+ cells from the PA and TIL (paired) samples $(n=3)$. Analyzed with paired Student's $t$ test. c Proportion of CD200R among CD4+ T cells between paired PA and TIL samples $(n=3)$. Analyzed with Student's $t$ test. d Proportion of CD200R in CD8+ $\mathrm{T}$ cells in the paired PA and TIL samples $(n=3)$. Analyzed with Student's $t$ test. PA, pulmonary artery; TIL, tumor-infiltrating leukocyte. database was introduced into this study. Based on the median CD200R mRNA (CD200R1) expression level, 517 samples were separated into the CD200R-high and CD200R-low groups. The mRNA expression levels of six immune checkpoint molecules, including PD-1(PDCD1), CTLA-4, TIM-3 (HAVCR2), lymphocyte-activation gene 3 (LAG-3), T cell immunoreceptor with Ig and ITIM do- mains (TIGIT), and indoleamine-pyrrole 2,3-dioxygenase, and the correlations of these molecules with CD200R1 were investigated. All six immune checkpoint molecules showed significantly higher expression levels in the CD200R-high group (Fig. 4). At the same time, the other immune-related genes also showed a similar tendency (online suppl. Fig. 4). 


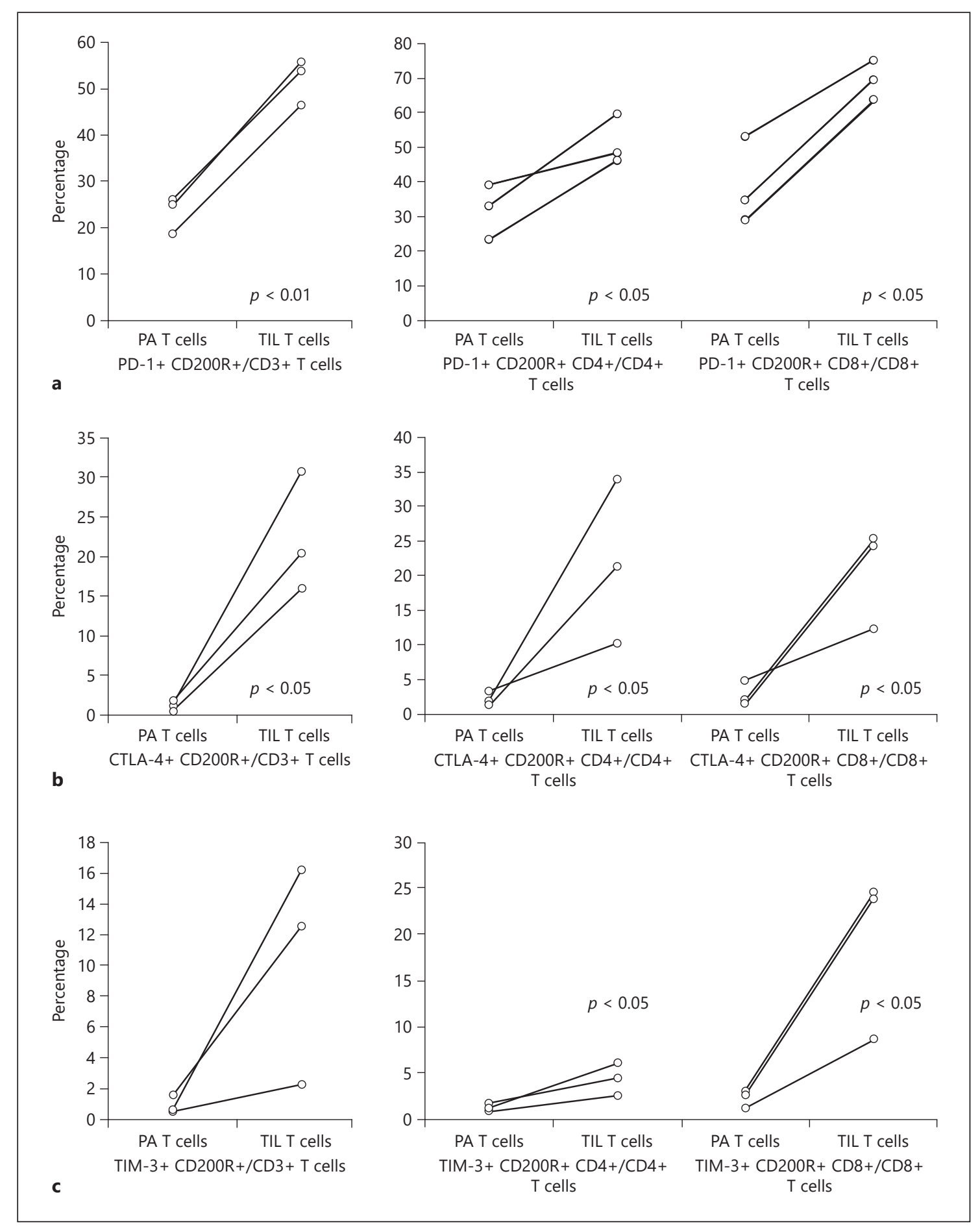

Fig. 3. Co-expression of other immune checkpoints in $\mathrm{T}$ cells. a Proportion of T cells co-expressing CD200R and the immune checkpoint PD-1 within CD3+ (left), CD4+, and CD8+ T cells (right) from the paired PA and TIL samples $(n=3)$. Analyzed with Student's $t$ test. b Proportion of T cells co-expressing CD200R and the immune checkpoint CTLA-4 within CD3+ (left), CD4+, and CD8+ T cells (right) from the paired PA and TIL samples $(n=3)$. Analyzed with Student's $t$ test. c Proportion of T cells co-express- ing CD200R and the immune checkpoint TIM-3 within CD3+ (left), CD4+, and CD8+ T cells (right) from the paired PA and TIL samples $(n=3)$. Analyzed with Student's $t$ test. CTLA-4, cytotoxic T-lymphocyte-associated protein 4; PA, pulmonary artery; PD-1, programmed cell death protein 1; TIL, tumor-infiltrating leukocyte; TIM-3, T cell immunoglobulin and mucin domain-containing protein 3 . 


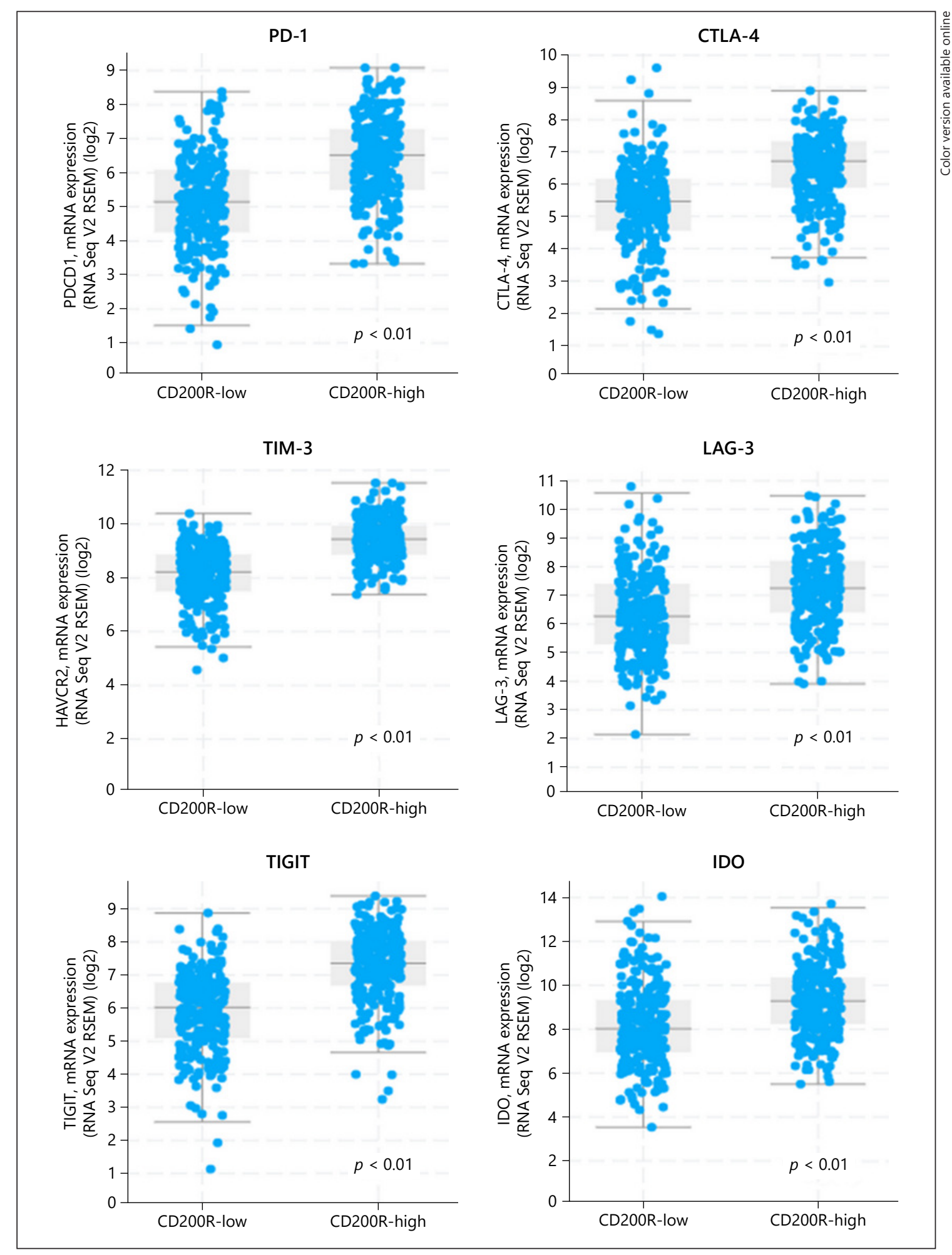

Fig. 4. Expression of CD200R in lung adenocarcinoma and its correlation with immune checkpoints according to public database analysis. Comparing the co-expression of mRNA between samples, separated according to medium expression value (CD200Rlow and CD200R-high group). Cohort: lung adenocarcinoma
(TCGA, Firehose Legacy), 517 samples derived from 516 patients. CTLA-4, cytotoxic T-lymphocyte-associated protein 4; IDO, indoleamine-pyrrole 2,3-dioxygenase; LAG-3, lymphocyte-activation gene 3; PD-1, programmed cell death protein 1; TIGIT, T cell immunoreceptor with Ig and ITIM domains. 


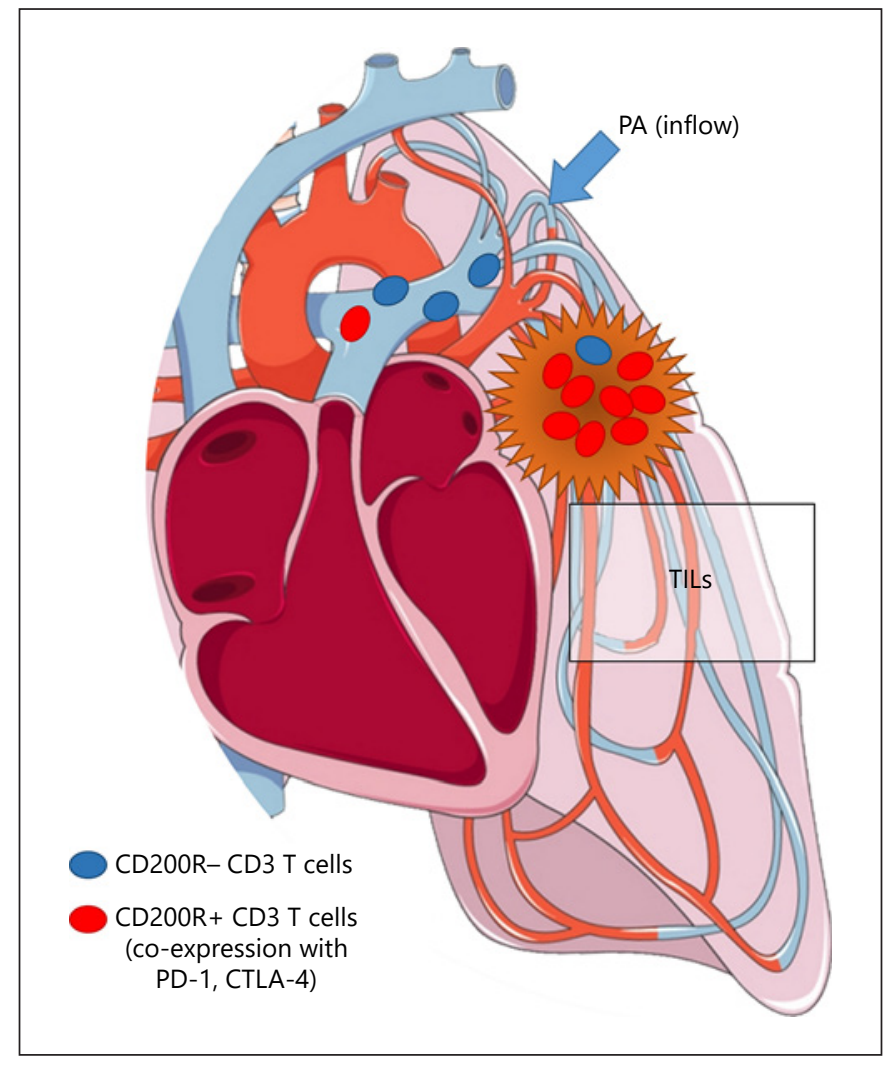

Fig. 5. Compared with the inflow from the PA, T cells within the cancer tissue show a significantly higher proportion of CD200R expression together with some other immune checkpoints, which suggests that a phenotypic change has occurred within the tumor microenvironment. CTLA-4, cytotoxic T-lymphocyte-associated protein 4; PA, pulmonary artery; PD-1, programmed cell death protein 1; TILs, tumor-infiltrating leukocytes.

\section{Discussion}

In this study, we demonstrated that CD200R was highly expressed in tumor-infiltrating T cells in NSCLC, and CD200R was highly co-expressed with multiple immune checkpoint molecules (Fig. 5). Most studies only focused on CD200R expression and its function among myeloid lineage cells $[5,7,18]$. However, our results demonstrated that both B and T cells express CD200R. Based on the current model of cancer-immune cycle [24], those T cells collected from cancer patients' PA were recruited from nearby lymph nodes and were activated by antigen-presenting cells. The PA blood used in the experiments was considered to be the inflow tract into the lung tissue, delivering blood circulating immune cells into the tumor. The significant differences of the immune checkpoint profile between the inflow tract of PA blood and accumulated tumor-infiltrating immune cells suggested that significant changes to their phenotype may have happened, and these changes might be due to the changes in the surrounding microenvironments, which is also reinforced by the results of public database analysis.

It is well reported that most tumor-infiltrating $\mathrm{T}$ cells have entered the stage of exhaustion or dysfunction [25]. Many studies have confirmed that the exhausted or dysfunctional $\mathrm{T}$ cells simultaneously express multiple immune checkpoint molecules, including PD-1, CTLA-4, TIM-3, LAG-3, and TIGIT [26-29]. Different levels of checkpoint co-expression represent highly heterogenic $\mathrm{T}$ cell populations with different stages of exhaustion, e.g., a subpopulation of $\mathrm{T}$ cells expressing both PD-1 and TIM-3 was well reported as a terminal exhausted group with no polyfunctionality retained [30-32]. It is widely accepted that before $\mathrm{T}$ cells differentiate into the terminal exhausted stage, dysfunction of $\mathrm{T}$ cells might be revised through the introduction of ICIs, such as anti-PD-1 [33], which can tip the balance of immune microenvironments from cold (inhibitory) to hot (activating). One previous study claimed that CD4 $\mathrm{T}$ cells increased CD200R expression level and loss of multifunctional potential during chronic infection [34], which might have happened similarly inside tumor tissue. The fact that CD200R is also largely co-expressed with other checkpoints inside tumors suggests that tumor-infiltrating $\mathrm{T}$ cells have entered the exhaustion stage. A combination of CD200R and other checkpoints might be able to act as a biomarker which can specify a subgroup of $\mathrm{T}$ cells with a certain level of exhaustion. Furthermore, a combinational blockage strategy of PD- 1 and CTLA- 4 has been designed, aiming to broaden the horizon for ICIs [35, 36]; targeting CD200R together with other checkpoints could be a promising candidate to restore $\mathrm{T}$ cell activities. Although an inhibitor of CD200 has currently entered into a clinical trial for chronic lymphocytic leukemia and multiple myeloma [37], the results of a lung cancer study claimed that CD200 might be a favorable prognostic factor [15]. Moreover, our previous study indicated that CD200 might potentially augment cancer cell sensitivity to chemotherapy $[17,38]$, which suggests that CD200 may potentially have functions other than immune modulation.

For revealing different stages of $\mathrm{T}$ cell infiltration and their connections to the CD200-CD200R axis, the better approach might be to construct a reliable model, such as a mouse or organoid model. Introducing a stabilized model will be helpful for downstream metabolic 
analysis and epigenetic studies that may further elucidate the subpopulations of CD200R+ T cells and exhausted cells. It also provides an option of inhibitor administration which can further confirm the mechanism of the CD200-CD200R axis and the potential of targeting CD200R.

In conclusion, our research investigated the expression level of an immune checkpoint, CD200R, in PA blood and TILs in NSCLC. The tumor-infiltrating T cells were more strongly dominated by CD200R-expressing T cells compared to the T cells from PA blood. Moreover, the expression of CD200R largely overlapped with that of other immune checkpoint molecules, including PD-1, CTLA-4, and TIM-3. Considering this finding, co-expression of CD200R and other immune checkpoints might be a good biomarker of $\mathrm{T}$ cell phenotypic changes or even a potential target for immune therapy.

\section{Acknowledgment}

We would like to thank Ms. Hiroko Hashimoto for her technical support. We also would like to show our appreciation to Dr. Naoya Sakamoto, Dr. Shingo Sakashita, Dr. Motohiro Kojima, and Dr. Satoshi Fujii for their helpful advice.

\section{Statement of Ethics}

This retrospective study was approved by the National Cancer Ethical Review Board (IRB approval number 2005-043).

\section{Conflict of Interest Statement}

No conflicts of interest are involved in this study.

\section{Funding Sources}

This work was supported in part by the National Cancer Center Research Fund (31-A-6).

\section{Author Contributions}

Y. Su: design, arrangement, conduction of the work, collection and analysis of all data, writing and editing of the manuscript including all pictures. S. Yamazaki and J. Suzuki: help with design. R. Morisue: helping with statistical analysis. T. Yoshikawa and T. Nakatsura: help with MACS techniques. M. Tsuboi: provision of all surgery specimens. A. Ochiai: help with design and statistical analysis. G. Ishii: coordination of the research, editing and revision of the manuscript.

\section{References}

1 Rittmeyer A, Barlesi F, Waterkamp D, Park K, Ciardiello F, von Pawel J, et al.; OAK Study Group. Atezolizumab versus docetaxel in patients with previously treated non-small-cell lung cancer $(\mathrm{OAK})$ : a phase 3, open-label, multicentre randomised controlled trial. Lancet. 2017 Jan;389(10066):255-65.

2 Herbst RS, Baas P, Kim DW, Felip E, PérezGracia JL, Han JY, et al. Pembrolizumab versus docetaxel for previously treated, PD-L1positive, advanced non-small-cell lung cancer (KEYNOTE-010): a randomised controlled trial. Lancet. 2016 Apr;387(10027):1540-50.

3 Rijkers ES, de Ruiter T, Baridi A, Veninga H, Hoek RM, Meyaard L. The inhibitory CD200R is differentially expressed on human and mouse $\mathrm{T}$ and $\mathrm{B}$ lymphocytes. Mol Immunol. 2008 Feb;45(4):1126-35.

4 Najar M, Raicevic G, Jebbawi F, De Bruyn C, Meuleman N, Bron D, et al. Characterization and functionality of the CD200-CD200R system during mesenchymal stromal cell interactions with T-lymphocytes. Immunol Lett. 2012 Aug;146(1-2):50-6.

5 Jenmalm MC, Cherwinski H, Bowman EP, Phillips JH, Sedgwick JD. Regulation of myeloid cell function through the CD200 receptor. J Immunol. 2006 Jan;176(1):191-9.

6 Gorczynski R, Chen Z, Kai Y, Lee L, Wong S, Marsden PA. CD200 is a ligand for all mem- bers of the CD200R family of immunoregulatory molecules. J Immunol. 2004 Jun;172(12): 7744-9.

7 Zhang S, Cherwinski H, Sedgwick JD, Phillips $\mathrm{JH}$. Molecular mechanisms of CD200 inhibition of mast cell activation. J Immunol. 2004 Dec;173(11):6786-93.

8 Prodeus A, Cydzik M, Abdul-Wahid A, Huang E, Khatri I, Gorczynski R, et al. Agonistic CD200R1 DNA Aptamers Are Potent Immunosuppressants That Prolong Allogeneic Skin Graft Survival. Mol Ther Nucleic Acids. 2014 Aug;3(8):e190.

9 Gorczynski RM, Chen Z, Lee L, Yu K, Hu J. AntiCD200R ameliorates collagen-induced arthritis in mice. Clin Immunol. 2002 Sep;104(3):256-64.

10 Prodeus A, Sparkes A, Fischer NW, Cydzik M, Huang E, Khatri I, et al. A Synthetic CrossSpecies CD200R1 Agonist Suppresses Inflammatory Immune Responses In Vivo. Mol Ther Nucleic Acids. 2018 Sep;12:350-8.

11 Liu JQ, Talebian F, Wu L, Liu Z, Li MS, Wu L, et al. A Critical Role for CD200R Signaling in Limiting the Growth and Metastasis of CD200+ Melanoma. J Immunol. 2016 Aug; 197(4):1489-97.

12 Erin N, Podnos A, Tanriover G, Duymus Ö, Cote E, Khatri I, et al. Bidirectional effect of CD200 on breast cancer development and metastasis, with ultimate outcome deter- mined by tumor aggressiveness and a cancerinduced inflammatory response. Oncogene. 2015 Jul;34(29):3860-70.

13 Huang S, Pan Y, Zhang Q, Sun W. Role of CD200/CD200R Signaling Pathway in Regulation of CD4+ T Cell Subsets During Thermal Ablation of Hepatocellular Carcinoma. Med Sci Monit. 2019 Mar;25:1718-28.

14 Zhang SS, Huang ZW, Li LX, Fu JJ, Xiao B. Identification of CD200+ colorectal cancer stem cells and their gene expression profile. Oncol Rep. 2016 Oct;36(4):2252-60.

15 Yoshimura K, Suzuki Y, Inoue Y, Tsuchiya K, Karayama M, Iwashita Y, et al. CD200 and CD200R1 are differentially expressed and have differential prognostic roles in nonsmall cell lung cancer. Oncoimmunology. 2020 Apr;9(1):1746554.

16 Blom LH, Martel BC, Larsen LF, Hansen CV, Christensen MP, Juel-Berg N, et al. The immunoglobulin superfamily member CD200R identifies cells involved in type 2 immune responses. Allergy. 2017 Jul;72(7):1081-90.

17 Ishibashi M, Neri S, Hashimoto H, Miyashita T, Yoshida T, Nakamura Y, et al. CD200-positive cancer associated fibroblasts augment the sensitivity of Epidermal Growth Factor Receptor mutation-positive lung adenocarcinomas to EGFR Tyrosine kinase inhibitors. Sci Rep. 2017 Apr;7(1):46662. 
18 Hoek RM, Ruuls SR, Murphy CA, Wright GJ, Goddard R, Zurawski SM, et al. Down-regulation of the macrophage lineage through interaction with OX2 (CD200). Science. 2000 Dec;290(5497):1768-71.

19 Wright GJ, Jones M, Puklavec MJ, Brown $\mathrm{MH}$, Barclay AN. The unusual distribution of the neuronal/lymphoid cell surface CD200 (OX2) glycoprotein is conserved in humans. Immunology. 2001 Feb;102(2):173-9.

20 Wright GJ, Cherwinski H, Foster-Cuevas M, Brooke G, Puklavec MJ, Bigler M, et al. Characterization of the CD200 receptor family in mice and humans and their interactions with CD200. J Immunol. 2003 Sep;171(6):303446.

21 Maeda R, Ishii G, Neri S, Aoyagi K, Haga H, Sasaki H, et al. Circulating CD14+CD204+ cells predict postoperative recurrence in nonsmall-cell lung cancer patients. J Thorac Oncol. 2014 Feb;9(2):179-88.

22 Cerami E, Gao J, Dogrusoz U, Gross BE, Sumer SO, Aksoy BA, et al. The cBio cancer genomics portal: an open platform for exploring multidimensional cancer genomics data. Cancer Discov. 2012 May;2(5):401-4.

23 Gao J, Aksoy BA, Dogrusoz U, Dresdner G, Gross B, Sumer SO, et al. Integrative analysis of complex cancer genomics and clinical profiles using the cBioPortal. Sci Signal. 2013 Apr;6(269):pl1.

24 Chen DS, Mellman I. Oncology meets immunology: the cancer-immunity cycle. Immunity. 2013 Jul;39(1):1-10.

25 Kataki A, Scheid P, Piet M, Marie B, Martinet $\mathrm{N}$, Martinet $\mathrm{Y}$, et al. Tumor infiltrating lymphocytes and macrophages have a potential dual role in lung cancer by supporting both host-defense and tumor progression. J Lab Clin Med. 2002 Nov;140(5):320-8.
26 Jin HT, Anderson AC, Tan WG, West EE, Ha SJ, Araki K, et al. Cooperation of Tim-3 and PD-1 in CD8 T-cell exhaustion during chronic viral infection. Proc Natl Acad Sci USA. 2010 Aug;107(33):14733-8.

27 Blackburn SD, Shin H, Haining WN, Zou T, Workman CJ, Polley A, et al. Coregulation of CD8+ T cell exhaustion by multiple inhibitory receptors during chronic viral infection. Nat Immunol. 2009 Jan;10(1):29-37.

28 Joller N, Hafler JP, Brynedal B, Kassam N, Spoerl S, Levin SD, et al. Cutting edge: TIGIT has T cell-intrinsic inhibitory functions. J Immunol. 2011 Feb;186(3):1338-42.

29 Barber DL, Wherry EJ, Masopust D, Zhu B, Allison JP, Sharpe AH, et al. Restoring function in exhausted CD8 $\mathrm{T}$ cells during chronic viral infection. Nature. 2006 Feb;439(7077): 682-7.

30 Paley MA, Kroy DC, Odorizzi PM, Johnnidis JB, Dolfi DV, Barnett BE, et al. Progenitor and terminal subsets of CD8+ T cells cooperate to contain chronic viral infection. Science. 2012 Nov;338(6111):1220-5.

31 Im SJ, Hashimoto M, Gerner MY, Lee J, Kissick HT, Burger MC, et al. Defining CD8+ T cells that provide the proliferative burst after PD-1 therapy. Nature. 2016 Sep;537(7620): $417-21$.

32 Miller BC, Sen DR, Al Abosy R, Bi K, Virkud YV, LaFleur MW, et al. Subsets of exhausted CD8+ T cells differentially mediate tumor control and respond to checkpoint blockade. Nat Immunol. 2019 Mar;20(3):326-36.
33 Thommen DS, Koelzer VH, Herzig P, Roller A, Trefny M, Dimeloe S, et al. A transcriptionally and functionally distinct PD-1+ CD8+ T cell pool with predictive potential in nonsmall-cell lung cancer treated with PD-1 blockade. Nat Med. 2018 Jul;24(7):994-1004.

34 Caserta S, Nausch N, Sawtell A, Drummond R, Barr T, Macdonald AS, et al. Chronic infection drives expression of the inhibitory receptor CD200R, and its ligand CD200, by mouse and human CD4 T cells. PLoS One. 2012; 7(4):e35466.

35 Duraiswamy J, Kaluza KM, Freeman GJ, Coukos G. Dual blockade of PD-1 and CTLA-4 combined with tumor vaccine effectively restores T-cell rejection function in tumors. Cancer Res. 2013 Jun;73(12):3591-603.

36 Huang RY, Francois A, McGray AR, Miliotto A, Odunsi K. Compensatory upregulation of PD-1, LAG-3, and CTLA-4 limits the efficacy of single-agent checkpoint blockade in metastatic ovarian cancer. Oncoimmunology. 2016 Oct;6(1):e1249561.

37 Mahadevan D, Lanasa MC, Farber C, Pandey M, Whelden M, Faas SJ, et al. Phase I study of samalizumab in chronic lymphocytic leukemia and multiple myeloma: blockade of the immune checkpoint CD200. J Immunother Cancer. 2019 Aug;7(1):227.

38 Lambrechts D, Wauters E, Boeckx B, Aibar S, Nittner D, Burton O, et al. Phenotype molding of stromal cells in the lung tumor microenvironment. Nat Med. 2018 Aug;24(8): 1277-89. 